\title{
Multifractal dimensions for random matrices, chaotic quantum maps, and many-body systems
}

\author{
Arnd Bäcker, ${ }^{1,2}$ Masudul Haque, ${ }^{1,3}$ and Ivan Khaymovich ${ }^{1}$ \\ ${ }^{1}$ Max-Planck-Institut für Physik komplexer Systeme, Nöthnitzer Straße 38, 01187 Dresden, Germany \\ ${ }^{2}$ Technische Universität Dresden, Institut für Theoretische Physik and Center for Dynamics, 01062 Dresden, Germany \\ ${ }^{3}$ Department of Theoretical Physics, Maynooth University, Co. Kildare, Ireland
}

(Dated: October 30, 2019)

\begin{abstract}
Multifractal dimensions allow for characterizing the localization properties of states in complex quantum systems. For ergodic states the finite-size versions of fractal dimensions converge to unity in the limit of large system size. However, the approach to the limiting behavior is remarkably slow. Thus, an understanding of the scaling and finite-size properties of fractal dimensions is essential. We present such a study for random matrix ensembles, and compare with two chaotic quantum systems - the kicked rotor and a spin chain. For random matrix ensembles we analytically obtain the finite-size dependence of the mean behavior of the multifractal dimensions, which provides a lower bound to the typical (logarithmic) averages. We show that finite statistics has remarkably strong effects, so that even random matrix computations deviate from analytic results (and show strong sample-to-sample variation), such that restoring agreement requires exponentially large sample sizes. For the quantized standard map (kicked rotor) the multifractal dimensions are found to follow the random matrix predictions closely, with the same finite statistics effects. For a XXZ spin-chain we find significant deviations from the random matrix prediction — the large-size scaling follows a system-specific path towards unity. This suggests that local many-body Hamiltonians are "weakly ergodic", in the sense that their eigenfunction statistics deviate from random matrix theory.
\end{abstract}

\section{INTRODUCTION}

Energy eigenstates are integral to the formulation of quantum mechanics. Except for especially simple systems, eigenstates usually are complicated objects, described in any basis by a large number of coefficients. Thus, it is natural to analyze eigenstate coefficients statistically. Statistical properties of eigenstates were investigated already very early in the context of transition strengths for complex nuclei [1] which can be described by random matrix ensembles [2], and are central to the study of quantum chaos, e.g., in quantum billiards [311] and in quantum maps [11-16]. They also play a crucial role in characterizing critical behaviors of Anderson transitions between localized and metallic phases in disordered systems [17]. Moreover, the properties of energy eigenstates are of particular importance for describing the behavior of isolated quantum many-body systems, e.g., concerning thermalization [18-23] and many-body localization [24-31].

The statistical properties of eigenstates have been characterized and studied in multiple ways. The distributions of eigenstates have been examined directly, e.g, for quantum billiards [4-10, 32-35], for many-body systems [34, 36], and for quantum maps [11-16] and random-matrix ensembles [3739]. The maxima of random waves and chaotic eigenstates have also been considered [15, 40]. Eigenstate statistics have often been characterized through the inverse participation ratio, extensively over several decades for single-particle systems [17, 41-44] and more recently also for many-body systems $[23,34,36,45-50]$. Generalizing the inverse participation ratio, eigenstate statistics has also been studied through the Shannon and Rényi entropies [45, 51-57]. Closely related to the Rényi entropies are the so-called fractal dimensions [58, 59], which are the topic of this work. Analysis of fractal dimensions ('multifractal analysis') is a standard tool in the study of (single-particle) Anderson localization [17] and has also been recently applied to eigenstates of many-body quantum systems [29, 34, 57, 60, 61].

If the $q$-th moment of the eigenstate coefficients scales like $N^{-(q-1) D_{q}^{\infty}}$ as a function of the Hilbert space dimension $N$, then the quantity $D_{q}^{\infty} \geq 0$ gives the (multi)fractal (Hausdorff) dimension of the corresponding support set in the limit $N \rightarrow \infty$. The fractal dimensions are particularly useful for distinguishing between localized and ergodic phases for single-particle lattice systems with disorder. The Andersonlocalized phase is characterized by zero fractal dimensions $D_{q}^{\infty}=0$ for $q>0$, as each eigenstate is localized at a finite number of sites. In contrast, so-called ergodic quantum eigenstates [17] are those states for which at least a finite fraction of the coefficients in the given basis contribute significantly, and thus $D_{q}^{\infty}=1$.

An important class of quantum systems are those with a well-defined classical limit showing chaotic dynamics in the sense that one has sensitive dependence on the initial conditions (positive Lyapunov exponents almost everywhere) and ergodicity (temporal averages of observables correspond to spatial averages for almost all initial conditions). In such cases one expects that the statistical properties of spectra can be described by those of corresponding random matrix ensembles [62-64]. In contrast, many-body systems usually do not have such a classical limit. We can define a many-body system as being "ergodic" or "chaotic" if the spectral statistics or eigenfunction statistics follow those of one of the random matrix ensembles. In either of these cases one expects in the large-size limit that the fractal dimensions of most eigenstates are equal to $D_{q}^{\infty}=1$ for all $q \geq 0$.

The fractal dimensions are of particular interest in characterizing multifractality, in which case $D_{q}^{\infty}$ has a nontrivial $q$-dependence, in contrast to ergodic (localized) states for which $D_{q}^{\infty}$ is equal to 1 (0) for all $q \geq 0$. Multifractal statistics appears at the Anderson localization transition for single-particle lattice systems [17, 65-71]. In addition, recent examples have reported (multi)fractal phases extend- 
ing over a whole range of parameters [72-86]. Multifractal wavefunctions have been found for some quantum maps $[68,70,87,88]$. For local many-body quantum Hamiltonians, the ground states have been found to display multifractal behavior, even in cases for which eigenstates at the center of the many-body spectrum show random-matrix behavior $[34,57,60,89-91]$. Also, the question of the existence of a multifractal phase in the vicinity of the many-body localization transition as well as its relation to the slow dynamical phases is under active debate $[31,57,61,73,74,92-96])$.

In this paper, we examine the finite-size dependence of fractal dimensions ( $N$-dependence of $D_{q}(N)$ ) for eigenstates of random matrices and of nominally chaotic systems. The eigenstates of these systems are expected to be at least weakly ergodic. Ergodic states are considered to be less exotic than multifractal states, since the large- $N$ limit is simple. However, we will present highly nontrivial scaling behaviors: $D_{q}(N)$ approaches unity extremely slowly and with large eigenstateto-eigenstate fluctuations. We will first present analytical and numerical results for the case of random-matrix ensembles, namely the circular orthogonal (COE) and unitary (CUE) ensembles. These results will then be compared to two physical systems which are expected to have ergodic behavior. The first is a paradigmatic model from quantum chaos: the quantum kicked rotor whose corresponding classical dynamics is given by the standard map. We will show that the multifractal properties of the quantized standard map with strongly chaotic classical dynamics follow the CUE predictions very closely. We then consider a non-integrable quantum spin chain. In this case the comparison is substantially more subtle because only the center of the many-body spectrum ("infinite-temperature" states) is expected to behave ergodically. We present numerical evidence that the behavior of many-body eigenstates is only "weakly ergodic", in the sense that $D_{q}(N)$ approaches unity for $N \rightarrow \infty$ but follows a different system-specific path compared to the COE case.

The paper is structured as follows. We introduce the fractal dimensions in Section II, in particular the mean and typical averages. In Section III we present analytic derivations for the random matrix ensembles and compare with numerical calculations for COE and CUE ensembles. In Section IV we present calculations of $D_{q}(N)$ for the chaotic quantum map and also compare with random matrix results Section $V$ treats as example of a many-body quantum system a spin chain in the chaotic regime, and an analysis of $D_{q}(N)$ is presented. In Section VI we summarize and point out open questions.

\section{FRACTAL DIMENSIONS}

To characterize the properties of a given state $\left|\Psi_{j}\right\rangle$ consider its expansion coefficients $c_{i}^{(j)}$ in some (finite) orthonormal basis $\left\{\left|\psi_{i}\right\rangle\right\}$, i.e. $\left|\Psi_{j}\right\rangle=\sum_{i=1}^{N} c_{i}^{(j)}\left|\psi_{i}\right\rangle$. Based on the moments

$$
I_{q}(j, N)=\sum_{i=1}^{N}\left|c_{i}^{(j)}\right|^{2 q}
$$

one defines the (finite- $N$ ) fractal dimensions for the given state

$$
\begin{aligned}
D_{q}(j, N) & =-\frac{1}{q-1} \frac{1}{\ln N} \ln I_{q}(j, N) \\
& =-\frac{1}{q-1} \frac{1}{\ln N} \ln \left(\sum_{i=1}^{N}\left|c_{i}^{(j)}\right|^{2 q}\right) .
\end{aligned}
$$

For fixed $N$ the fractal dimensions are monotonically decreasing functions of $q$ with $0 \leq D_{q}(N) \leq 1$ for $q \geq 0$. In the limit $q \rightarrow 1$ one gets by l'Hôpital's rule the Shannon information dimension

$$
D_{1}(j, N)=-\frac{1}{\ln (N)} \sum_{i=1}^{N}\left|c_{i}^{(j)}\right|^{2} \ln \left|c_{i}^{(j)}\right|^{2} .
$$

One may now consider an average over an ensemble of states, which is denoted by

$$
D_{q}(N)=\left\langle D_{q}(j, N)\right\rangle \equiv-\frac{1}{q-1} \frac{1}{\ln N}\left\langle\ln I_{q}(j, N)\right\rangle .
$$

Finally, the fractal dimensions $D_{q}^{\infty}$ are defined in the limit $N \rightarrow \infty$ [97], i.e.

$$
D_{q}^{\infty} \equiv \lim _{N \rightarrow \infty} D_{q}(N)
$$

If $D_{q}^{\infty}$ depends on $q>0$ in a nontrivial way, the states are multifractal. For constant $D_{q}^{\infty}<1$ the states are fractal, ergodic behavior corresponds to $D_{q}^{\infty}=1$, and localized states correspond to $D_{q}^{\infty}=0$.

Thus the fractal dimensions $D_{q}^{\infty}$ describe the asymptotic scaling behavior of the moments of typical eigenstates as $N \rightarrow \infty$, i.e.

$$
\begin{aligned}
\left\langle I_{q}(j, N)\right\rangle_{\mathrm{typ}} & \equiv \exp \left[\left\langle\ln I_{q}(j, N)\right\rangle\right] \\
& \stackrel{N \rightarrow \infty}{\sim} N^{-D_{q}^{\infty}(q-1)} .
\end{aligned}
$$

Numerically, $D_{q}^{\infty}$ can only be estimated by extrapolating the results of finite- $N$ computations using Eq. (5).

The leading size-dependence of $D_{q}(N)$ is often of the form

$$
D_{q}(N) \sim D_{q}^{\infty}-f_{q} / \ln N,
$$

so that using Eq. (5) the moments can be written as

$$
\left\langle I_{q}(j, N)\right\rangle_{\mathrm{typ}}=N^{-D_{q}(N)(q-1)} \simeq c_{q} N^{-D_{q}^{\infty}(q-1)},
$$

with $c_{q}=\mathrm{e}^{(q-1) f_{q}}$. When the finite-size correction to $D_{q}^{\infty}$ is not exactly or solely of the form proportional to $1 / \ln N$, the pre-factor $c_{q}$ acquires a weak dependence on $N$.

Random matrix theory allows for a universal description of the statistical properties of ergodic eigenstates in many different situations. Thus it should also provide a prediction for the finite- $N$ scaling of $D_{q}(N)$, where the average in Eq. (5) is performed over a suitable random matrix ensemble. However, an analytical computation of the ensemble average in Eq. (5) over the logarithm of the moments is a daunting task. 
Thus instead we will use the ensemble averaged moments $\widetilde{I}_{q}(N) \equiv\left\langle I_{q}(j, N)\right\rangle$ and take the logarithm afterwards, i.e.

$$
\widetilde{D}_{q}(N)=-\frac{1}{q-1} \frac{1}{\ln N} \ln \widetilde{I}_{q}(N) .
$$

By Jensen's inequality $\widetilde{D}_{q}(N)$ provides a lower bound to $D_{q}(N)$,

$$
\widetilde{D}_{q}(N) \leq D_{q}(N)
$$

as the logarithm is a concave function. In particular $\widetilde{D}_{q}(N) \rightarrow$ 1 implies $D_{q}(N) \rightarrow 1$.

\section{RANDOM MATRIX PREDICTIONS}

As specific random matrix ensembles we consider the circular unitary ensemble (CUE) of complex unitary matrices, describing systems without any antiunitary symmetries and the circular orthogonal ensemble $(\mathrm{COE})$ of real orthogonal matrices, describing systems with one antiunitary symmetry, e.g. time-reversal. Note that the results for the eigenvector statistics of the CUE and COE also apply to the Gaussian unitary ensemble (GUE) and the Gaussian orthogonal ensemble (GOE), respectively.

\section{A. Circular orthogonal ensemble}

For the COE the eigenvectors can be chosen to be real and the only requirement for the coefficients $c_{i}^{(j)}$ is the normalization

$$
\sum_{i=1}^{N}\left(c_{i}^{(j)}\right)^{2}=1
$$

This condition implies that the probability density of one (rescaled) component $N\left(c_{i}^{(j)}\right)^{2}$ to have a specific value $\eta$ is given by [1, Eq. (7.5)]

$$
P_{N}^{\mathrm{COE}}(\eta)=\frac{1}{\sqrt{\pi N \eta}} \frac{\Gamma(N / 2)}{\Gamma((N-1) / 2)}(1-\eta / N)^{(N-3) / 2} .
$$

The corresponding eigenfunction moments (1) calculated from this distribution are

$$
\widetilde{I}_{q}^{\mathrm{COE}}(N)=\frac{N}{\sqrt{\pi}} \frac{\Gamma(N / 2) \Gamma(q+1 / 2)}{\Gamma(q+N / 2)} .
$$

For large $N$ one gets

$$
\begin{aligned}
\widetilde{I}_{q}^{\mathrm{COE}}(N) \simeq & \frac{\Gamma(q+1 / 2)(2 \mathrm{e})^{q}}{\sqrt{\pi}} N^{-(q-1)} \\
& \times\left(1-\frac{2}{N}\right)^{\frac{N-1}{2}}\left(1-\frac{2(q-1)}{N}\right)^{q+\frac{N-1}{2}} \\
\simeq & \frac{\Gamma(q+1 / 2) 2^{q}}{\sqrt{\pi}} N^{-(q-1)}+O\left(N^{-q}\right) \\
& \sim N^{-(q-1)}
\end{aligned}
$$

Note that one obtains from (13) in the limit of large $N$ the so-called Porter-Thomas distribution [98]

$$
P^{\mathrm{COE}}(\eta)=\frac{1}{\sqrt{2 \pi \eta}} \exp (-\eta / 2)
$$

Based on the moments (14), inserted in Eq. (10), one gets the COE prediction for the finite- $N$ scaling of the fractal dimensions

$$
\begin{aligned}
\widetilde{D}_{q}^{\mathrm{COE}}(N) & =-\frac{1}{(q-1) \ln N} \ln \left(\widetilde{I}_{q}^{\mathrm{COE}}(N)\right) \\
& =-\frac{1}{(q-1) \ln N} \ln \left(\frac{N \Gamma(N / 2) \Gamma(q+1 / 2)}{\sqrt{\pi} \Gamma(q+N / 2)}\right) \\
& \simeq 1-\frac{1}{(q-1) \ln N} \ln \left(\frac{\Gamma(q+1 / 2) 2^{q}}{\sqrt{\pi}}\right)
\end{aligned}
$$

For $q=1$ this gives $\widetilde{D}_{1}^{\mathrm{COE}}(N)=1-(\ln 2+\psi(3 / 2)) / \ln N$, with the digamma function $\psi(x)=\Gamma^{\prime}(x) / \Gamma(x)$ [99, 5.2.E2]. The fractal dimensions approach $\widetilde{D}_{q}^{\mathrm{COE}}(N) \rightarrow 1$ with logarithmic corrections $\sim 1 / \ln N$. For the pre-factor $c_{q}$ intro-
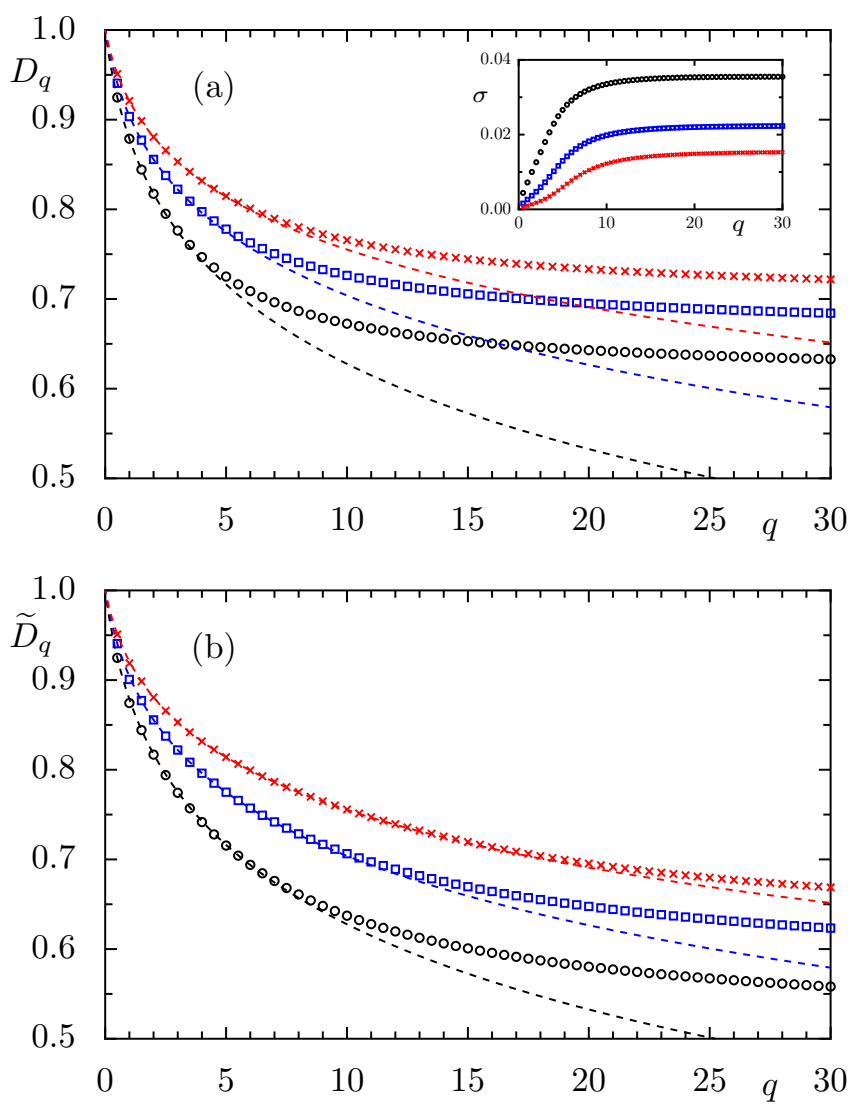

FIG. 1. Fractal dimensions (a) $D_{q}(N)$ of typical and (b) $\widetilde{D}_{q}(N)$ of mean eigenstate moments for the COE for $N=400,2000,10000$ (black circles, blue squares, red crosses) in comparison with $\widetilde{D}_{q}^{\mathrm{COE}}(N)$, Eq. (21), dashed lines. The inset in (a) shows the standard deviation $\sigma(q)$ of the fluctuations of $D_{q}(j, N)$ around $D_{q}(N)$. 
duced in Eq. (9), this gives

$$
c_{q}^{\mathrm{COE}} \simeq \Gamma(q+1 / 2) 2^{q} / \sqrt{\pi} .
$$

Figure 1(a) shows $D_{q}(N)$ for $N=400,2000$, and 10000, each computed from one realization of the COE, numerically generated as described in [100]. The curves are still very far from $D_{q}=1$, but a slow logarithmic approach with increasing $N$ is clearly seen. The analytical result $\widetilde{D}_{q}^{\mathrm{COE}}(N)$, Eq. (21), provides according to the inequality (11), a lower bound. This bound even gives a good approximation up to some value of $q$, which increases with increasing $N$. In Fig. 1(b) we show $\widetilde{D}_{q}(N)$ for the COE, i.e. for one realization the moments $\widetilde{I}_{q}^{\mathrm{COE}}(N)$ are computed and then Eq. (10) is used. The agreement with the analytical result $\widetilde{D}_{q}^{\mathrm{COE}}(N)$, Eq. (21), is much better. However, for larger values of $q$, there are still prominent deviations from the analytic predictions. We will discuss the origin of these deviations in Sec. III C.

The inset in Fig. 1(a) shows the standard deviation $\sigma(q)$ of the fluctuations of $D_{q}(j, N)$ around $D_{q}(N)$. For fixed $N$, the state-to-state fluctuations increase with increasing $q$, and appear to eventually saturate. Larger values of $N$ lead to smaller fluctuations.

\section{B. Circular unitary ensemble}

For the CUE the eigenvectors are complex, fulfilling the normalization condition

$$
\sum_{i=1}^{N}\left|c_{i}^{(j)}\right|^{2}=1
$$

This implies that the probability density of one (rescaled) component $N\left|c_{i}^{(j)}\right|^{2}$ to have a specific value $\eta$ is given by [14]

$$
P_{N}^{\mathrm{CUE}}(\eta)=(1-1 / N)(1-\eta / N)^{N-2}
$$

The corresponding eigenfunction moments (1) calculated from this distribution are

$$
\widetilde{I}_{q}^{\mathrm{CUE}}(N)=\frac{q ! N !}{(N+q-1) !} .
$$

For large $N$ one gets

$$
\begin{aligned}
\widetilde{I}_{q}^{\mathrm{CUE}}(N) \simeq & q ! \mathrm{e}^{q} N^{-(q-1)} \\
& \times\left(1-\frac{1}{N}\right)^{N-\frac{1}{2}}\left(1-\frac{q-1}{N}\right)^{q+N-\frac{1}{2}} \\
\simeq & q ! N^{-(q-1)}+O\left(N^{-q}\right) \\
\sim & N^{-(q-1)} .
\end{aligned}
$$

Note that one obtains from Eq. (25), in the limit of large $N$,

$$
P^{\mathrm{CUE}}(\eta)=\exp (-\eta)
$$

Based on the moments (26), inserted in Eq. (10), one gets the CUE prediction for the $N$-dependence of the fractal dimensions

$$
\begin{aligned}
\widetilde{D}_{q}^{\mathrm{CUE}}(N) & =-\frac{1}{(q-1) \ln N} \ln \left(N \widetilde{I}_{q}^{\mathrm{CUE}}(N)\right) \\
& =-\frac{1}{(q-1) \ln N} \ln \left(\frac{q ! N !}{(N-1+q) !}\right) \\
& \simeq 1-\frac{\ln (q !)}{(q-1) \ln N} .
\end{aligned}
$$

For $q=1$ this gives $\widetilde{D}_{1}^{\mathrm{CUE}}(N)=1-(1-\gamma) / \ln N$, where $\gamma \simeq 0.577216$ is Euler's constant. Clearly, as $N \rightarrow \infty$ the fractal dimension approaches $\widetilde{D}_{q}^{\mathrm{CUE}}(N) \rightarrow 1$ with logarithmic corrections $\sim 1 / \ln N$, as in the COE case. For the prefactor $c_{q}$ introduced in Eq. (9), this gives

$$
c_{q}^{\mathrm{CUE}} \simeq q !
$$

Figure 2(a) shows a comparison of $D_{q}(N)$ for the CUE with the lower-bound $\widetilde{D}_{q}^{\mathrm{CUE}}(N)$, Eq. (33) for $N=400,2000$ and 10000 , each using one realization. Similarly to the case
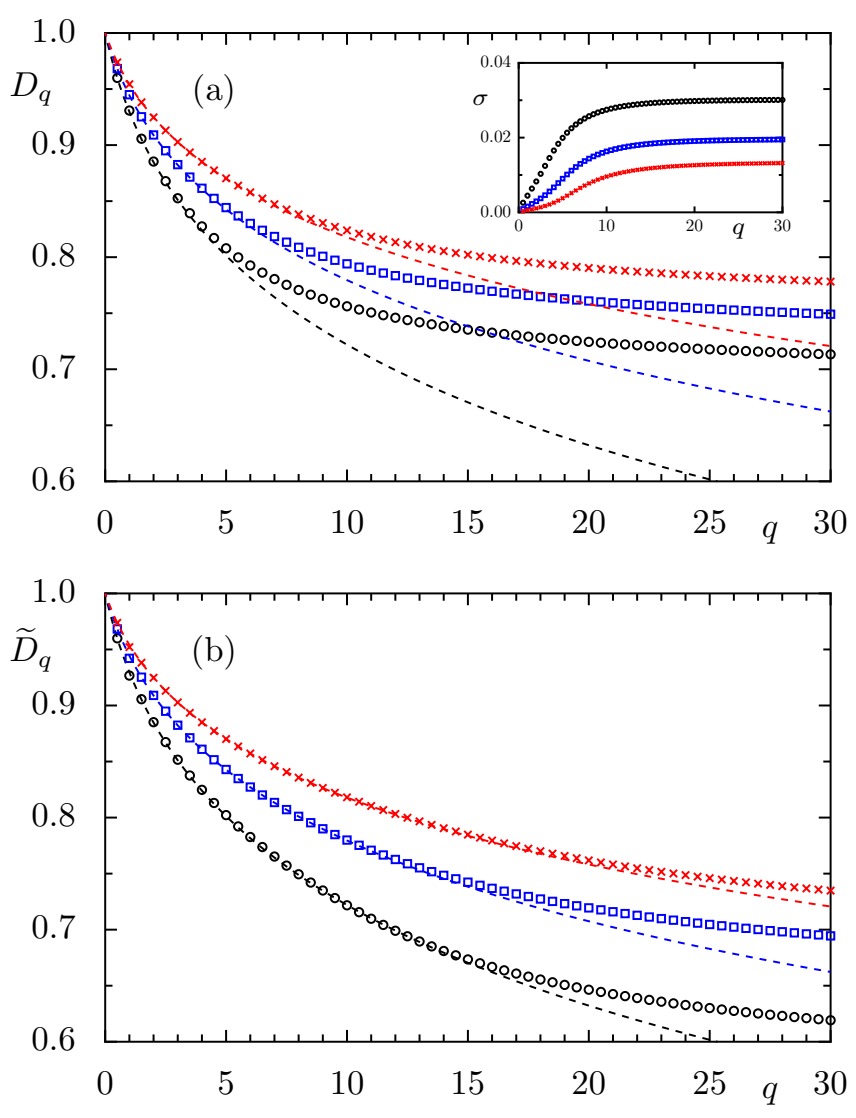

FIG. 2. Fractal dimensions (a) $D_{q}(N)$ of typical and (b) $\widetilde{D}_{q}(N)$ of mean eigenstate moments for the CUE for $N=400,2000,10000$ (black circles, blue squares, red crosses) in comparison with $\widetilde{D}_{q}^{\text {CUE }}(N)$, Eq. (33), dashed lines. The inset in (a) shows the standard deviation $\sigma(q)$ of the fluctuations of $D_{q}(j, N)$ around $D_{q}(N)$. 

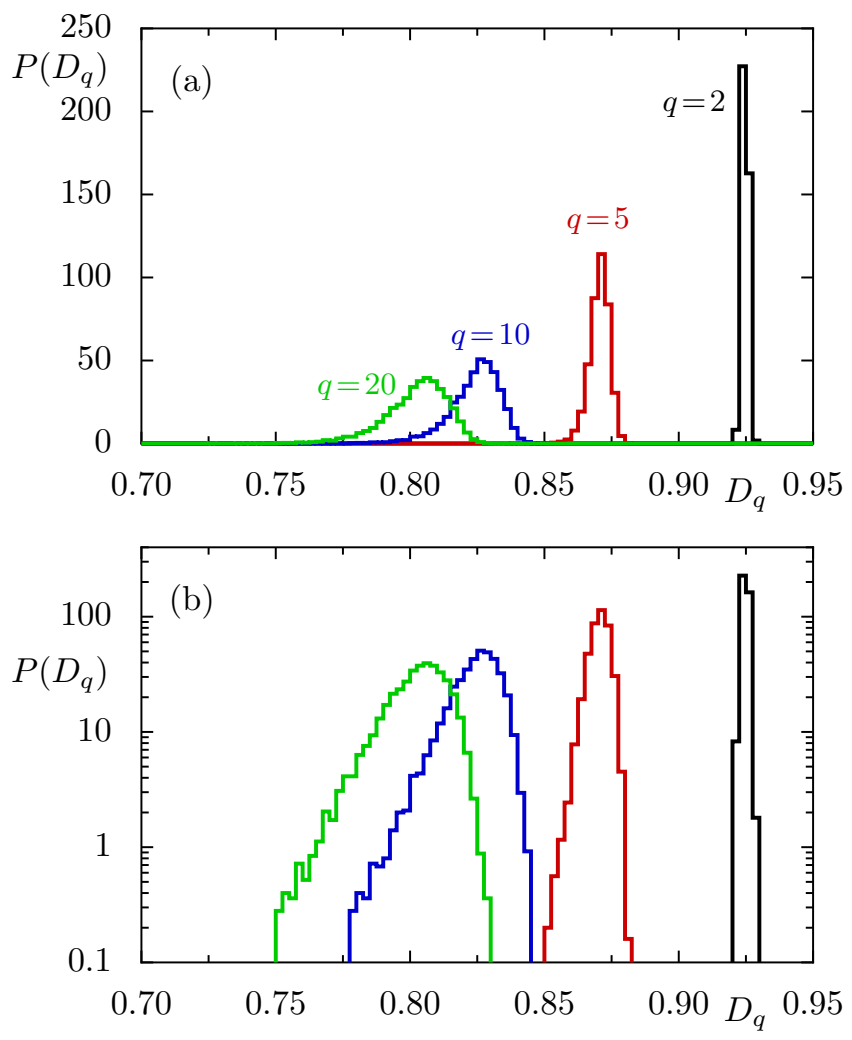

FIG. 3. (a) Distributions $P\left(D_{q}(j, N)\right)$ for one realization of the CUE for $N=10000$ and $q=2,5,10,20$. (b) Semi-logarithmic representation.

of the COE one finds an overall slow logarithmic approach towards $D_{q}=1$ with increasing $N$. The deviations from the lower bound $\widetilde{D}_{q}^{\mathrm{CUE}}(N)$, Eq. (33) start for larger $q$ with increasing $N$ than for the COE. Also for $\widetilde{D}_{q}(N)$ for the CUE, shown in Fig. 2(b), the agreement with the analytical result $\widetilde{D}_{q}^{\mathrm{CUE}}(N)$, Eq. (33) is better, however, again with unexpected prominent deviations for larger $q$.

In Figs. 1(a) and 2(a), we have presented $D_{q}(N)$, which is the average of $D_{q}(j, N)$ over $N$ states. However, there is quite a variation in the values of $D_{q}(j, N)$ themselves. This is already indicated by the standard deviation $\sigma(q)$, as shown in the insets Fig. 1(a) and Fig. 2(a) but better seen in the full distribution of $D_{q}(j, N)$. Figure 3(a) shows the histograms for $D_{q}(j, N)$ for the CUE for $N=10000$ and $q=2,5,10,20$. With increasing $q$, the mean decreases while the variance increases. This can also be understood intuitively, as larger values of $q$ correspond to higher moments of the eigenstate coefficients which therefore emphasizes the tails of the distribution of the coefficients. The semi-logarithmic representation in Fig. 3(b) shows that the tails towards smaller $D_{q}$ become approximately a straight line, i.e. show exponential behavior, while the tails towards larger $D_{q}$ are close to a Gaussian decay.

Based on the properties of the distributions $P\left(D_{q}(j, N)\right)$ one can draw several conclusions about the behavior of $D_{q}(N)$ and $\widetilde{D}_{q}(N)$ : (i) The fact that $P\left(D_{q}(j, N)\right)$ has a rapid decay in both directions ensures that the values of $D_{q}(N)$ and $\widetilde{D}_{q}(N)$ have similar orders of magnitude, as observed in Figs. 1 and 2. (ii) However, for larger $q$ the numerically computed $\widetilde{D}_{q}(N)$ are always above the analytical results $\widetilde{D}_{q}^{\mathrm{COE}}(N)$ and $\widetilde{D}_{q}^{\mathrm{CUE}}(N)$, see Figs. 1(b) and 2(b). The origin for this is the skewness of the distributions $P\left(D_{q}(j, N)\right)$ towards lower values of $D_{q}(j, N)$ together with the monotonic decay of the function $N^{-(q-1) D_{q}}$ with $D_{q}$ at $q>1$. Indeed, the analytical results $\widetilde{D}_{q}^{\mathrm{COE}}(N)$ and $\widetilde{D}_{q}^{\mathrm{CUE}}(N)$ can also be obtained by the integral

$$
N^{-(q-1) \widetilde{D}_{q}(N)}=\int P\left(D_{q}\right) N^{-(q-1) D_{q}} d D_{q},
$$

where the integrand is more skewed to the left in comparison with $P\left(D_{q}\right)$, but still decays rapidly. The numerically sampled $\widetilde{D}_{q}(N)$ is governed by the most probable values around the maximum of the integrand in (36). Thus it deviates to larger values from $\widetilde{D}_{q}^{\mathrm{COE}}(N)$. (iii) The shape of the distribution of $P\left(D_{q}\right)$ appears to stabilize with increasing $q$; see for example the $q=10$ and $q=20$ distributions in Fig. 3. This corresponds to the saturation of the standard deviation $\sigma(q)$ at large $q$, seen in the insets of Figs. 1(a) and 2(a)

\section{Finite-statistics corrections}

In this section we consider corrections to the moments $\widetilde{I}_{q}(N)$ and fractal dimensions $D_{q}(N)$ and $\widetilde{D}_{q}(N)$ due to finite statistics. This allows to estimate the value of $q$ above which the numerical calculations deviate from analytic predictions.

We consider a situation where one obtains the data from a finite number $N_{r}$ of eigenstates, which may be from one or several (e.g. disorder) realizations. Statistical errors come into play because of the finiteness of $N_{r}$. We can characterize these errors by considering how the distribution $P\left(N\left|c_{i}^{(j)}\right|^{2}=\right.$ $\eta) \equiv P(\eta)$ is numerically approximated by a histogram. The histogram is normalized by $N_{r}$ and has bin sizes $\Delta \eta$. We first consider the bin sizes $\Delta \eta$ to be independent of $\eta$. At the edge of the distribution, i.e. for larger values of $\eta$, the number of counts per bin is smaller, and hence statistically less reliable. When there are only a few counts, $C \sim O(1)$, statistical errors become significant. The bin at which this occurs, i.e., the value $\eta=\eta^{*}$, is given by the condition

$$
N_{r} P\left(\eta^{*}\right) \Delta \eta \simeq C .
$$

For the CUE case, using the exponential (31) as large- $N$ approximation, one obtains the condition

$$
\eta^{*}\left(N_{r}\right) \simeq \ln \left[\frac{N_{r} \Delta \eta}{C}\right]=\ln \bar{N}_{r}
$$

where $\bar{N}_{r} \equiv\left(N_{r} \Delta \eta / C\right)$.

For the COE case, the large- $N$ approximation is the PorterThomas distribution (19). For this we cannot solve Eq. (37) for $\eta^{*}$ in closed form, but approximating iteratively, we obtain

$$
\begin{aligned}
\eta^{*}\left(N_{r}\right) & \simeq 2 \ln \bar{N}_{r}+\ln \eta \\
& =2 \ln \bar{N}_{r}+\ln \left[2 \ln \bar{N}_{r}+\ln \eta\right]=\ldots,
\end{aligned}
$$


In the iterative solution for the COE case, the corrections to the leading term are either constant or multiple-logarithmic functions of $\bar{N}_{r}$; for our estimate we neglect these weakly varying functions and keep only the leading $\left(2 \ln \bar{N}_{r}\right)$ term. Thus we get

$$
\eta^{*}\left(N_{r}\right) \simeq \frac{2}{\beta} \ln \bar{N}_{r} \simeq \frac{2}{\beta} \ln N_{r},
$$

up to $O(1)$ constants and additive weaker functions of $N_{r}$. Here $\beta=1$ for the $\mathrm{COE}$ and $\beta=2$ for the CUE.

Note that there is no fundamental reason for the binning to be linear, i.e., for $\Delta \eta$ to be independent of $\eta$. If one uses logarithmic binning, $\Delta \eta \propto \eta$, one obtains corrections to the above estimate which are of double-logarithmic form, and hence can be neglected as done above.

To obtain an estimate for the value $q^{*}$ of $q$ at which statistical errors become significant, we have to to relate $q$ to $\eta$. Writing the $q$-th moment (1) as

$$
\begin{aligned}
N^{q-1}\left\langle I_{q}(j, N)\right\rangle & =\int_{0}^{N} \mathrm{~d} \eta \eta^{q} P(\eta) \\
& =\int_{0}^{N} \mathrm{~d} \eta \mathrm{e}^{q \ln \eta+\ln P(\eta)},
\end{aligned}
$$

we use the saddle point approximation to note that the main contribution comes from the value of $\eta$ that maximizes the exponent:

$$
\begin{aligned}
0=\frac{\mathrm{d}}{\mathrm{d} \eta}[q \ln \eta+\ln P(\eta)]=\frac{q}{\eta} & +\frac{\mathrm{d} \ln P(\eta)}{\mathrm{d} \eta} \\
& =\frac{q}{\eta}-\frac{\beta}{2}+\frac{\beta-2}{2 \eta} .
\end{aligned}
$$

Thus the main contribution to the $q$-th moment comes from $\eta(q)=(2 q+\beta-2) / \beta$. When $q$ gets so large that this $\eta(q)$ exceeds $\eta^{*}$, statistical errors become significant. Thus the value of $q$ beyond which statistical errors are significant is

$$
q^{\star}\left(N_{r}\right)=\frac{\beta}{2} \eta^{\star}\left(N_{r}\right)+1-\frac{\beta}{2} \simeq \ln N_{r} .
$$

This estimate neglects $O(1)$ constants and weaker (doublelogarithmic) dependences on $N_{r}$. In addition, the argument relies on some constants that cannot by nature be firmly specified, such as the bin count $C$ at which we consider statistical errors to become significant. Finally, the deviation between numerical and analytical predictions, seen in Figs. 1 and 2, gradually increase with $q$ and do not start at a sharply defined value of $q^{*}$. For all these reasons, we do not expect the estimate to be quantitatively accurate.

Figure 4 shows numerical estimates of $q^{\star}(N)$ as a function of $\ln N$. This gives an idea of how well the data for $D_{q}(N)$ and $\widetilde{D}_{q}(N)$ for one realization of the CUE and the COE are described by $\widetilde{D}_{q}^{\mathrm{CUE}}(N)$, Eq. (21), and $\widetilde{D}_{q}^{\mathrm{COE}}(N)$, Eq. (33), respectively. We determine $q^{\star}(N)$ as the lowest value of $q$ for which $D_{q}(N)$ or $\widetilde{D}_{q}(N)$ differ from the random matrix prediction by more than $f=0.009$, i.e.

$$
\left|D_{q}(N)-D_{q}^{\mathrm{RMT}}(N)\right| \leq f \quad \text { for } q<q^{\star}(N) .
$$

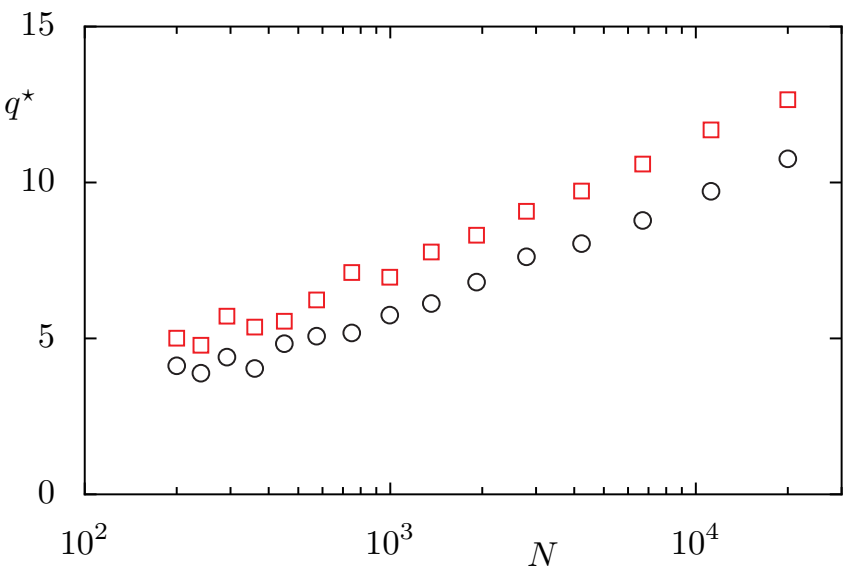

FIG. 4. Plot of the moment $q^{\star}(N)$ from which on RMT prediction and data beginn to differ by more than $f=0.009$; COE (black circles) and CUE (red squares).

Here $f=0.009$ approximately corresponds to the vertical extent of the symbols in Figs. 1 and 2. Of course, this estimate will depend on the choice of $f$, which is arbitrary. Despite this uncertainty and those discussed above, an approximate straight-line dependence is observed, i.e.

$$
q^{\star}(N) \sim \ln N
$$

in agreement with the theoretical expectation (45) for $N_{r}=$ $N$.

\section{CHAOTIC QUANTUM MAP}

For quantum systems whose corresponding classical dynamics is fully chaotic one expects that the statistics of eigenvalues and eigenstates can be described by random matrix theory. Still, even if the spectral statistics, e.g. for the levelspacing distribution, follow the corresponding random matrix results, this need not hold equally well for the statistics of eigenstates. Thus we now investigate, starting with a singleparticle system, how well the results for the scaling of the fractal dimensions are fulfilled for different types of chaotic quantum systems. In particular deviations may reveal interesting physics.

As a prototypical example of a system with chaotic classical dynamics we consider a time-periodically kicked system whose Hamiltonian reads

$$
H(x, p, t)=\frac{1}{2} p^{2}+V(x) \sum_{n=-\infty}^{\infty} \delta(t-n) .
$$

Here the sum describes a periodic sequence of kicks with unit time as kicking period. For $V(x)=\frac{K}{4 \pi^{2}} \cos (2 \pi x)$ one obtains the so-called kicked rotor. Its stroboscopic dynamics considered before consecutive kicks, gives the area-preserving standard map [101], $(x, p) \mapsto\left(x^{\prime}, p^{\prime}\right)$,

$$
\begin{aligned}
& x^{\prime}=x+p^{\prime} \\
& p^{\prime}=p+\frac{K}{2 \pi} \sin (2 \pi x),
\end{aligned}
$$


for which we consider $x, p \in[0,1[$ with periodic boundary conditions so that the phase space is a two-dimensional torus. For sufficiently large kicking strength $K$ the standard map is strongly chaotic [101, 102]. As example we use $K=9$, see the inset in Fig. 5, for which numerically no regular islands on any relevant scales have been found.

Quantum mechanically, the torus phase space leads to a finite Hilbert space of dimension $N$, see e.g. Refs. [103-107]. The effective Planck constant is $h=1 / N$ and $N \rightarrow \infty$ corresponds to the semiclassical limit. The quantum time evolution between consecutive kicks is given by a unitary timeevolution operator which can be represented in position space by a matrix with elements

$$
\begin{aligned}
U\left(n^{\prime}, n\right)= & \frac{1}{N} \exp \left(-\mathrm{i} N \frac{K}{2 \pi} \cos \left(\frac{2 \pi}{N}(n+\alpha)\right)\right) \\
\times & \sum_{m=0}^{N-1} \exp \left(-\frac{\pi \mathrm{i}}{N}(m+\beta)^{2}\right) \\
& \times \exp \left(\frac{2 \pi \mathrm{i}}{N}(m+\beta)\left(n-n^{\prime}\right)\right),
\end{aligned}
$$

where $n, n^{\prime} \in\{0,1, \ldots, N-1\}$. Thus one gets the eigenvalue problem

$$
U\left|\psi_{j}\right\rangle=\mathrm{e}^{\mathrm{i} \varphi_{n}}\left|\psi_{j}\right\rangle,
$$

with eigenphases $\varphi_{n} \in[0,2 \pi[$ as all eigenvalues lie on the unit circle due to the unitarity of $U$.

The quantum phases $\beta$ and $\alpha$ in Eq. (51) determine the boundary conditions due to the periodicity in position and momentum, respectively. Choosing $(\alpha, \beta)=(0.2,0.24)$ ensures that both time reversal symmetry and parity are broken, so that the consecutive level spacing distribution of this quantized standard map follows the prediction for the CUE.

Figure 5 shows a comparison of $D_{q}(N)$, computed from one realization of the quantized standard map for different $N=400,2000$, and 10000, with the lower-bound $\widetilde{D}_{q}^{\mathrm{CUE}}(N)$, Eq. (33). The agreement with the CUE results of Fig. 2(a) is quite good, and improves with increasing $N$. Thus overall one can conclude from Fig. 5 that the multifractal moments of the eigenvectors of the quantized standard map with fully chaotic dynamics are very well described by the corresponding random matrix computations. For the CUE and the quantized standard map, the $\widetilde{D}_{q}(N)$ show similar deviations from the analytical prediction (33).

To analyze the scaling of $D_{q}(N)$ and $\widetilde{D}_{q}(N)$ towards 1 in the limit $N \rightarrow \infty$, Fig. 6 shows the fractal dimensions vs. $1 / \ln N$. The comparison with $\widetilde{D}_{q}^{\mathrm{CUE}}(N)$, Eq. (33), and the results for the CUE, displayed in Fig. 6(b), show a similar scaling. For larger values of $q$ the fluctuations become more pronounced. For $D_{q}(N)$, displayed in Fig. 6(a), there is good agreement with the lower bound provided by $\widetilde{D}_{q}^{\mathrm{CUE}}(N)$, Eq. (33) when $q$ is small. However for larger values of $q$ there are clear deviations of the fractal dimensions. With increasing $N$, i.e. decreasing $1 / \ln N$, the data approach the lower bound (33) from above. Moreover the numerical results show that the finite-size corrections both for $D_{q}(N)$ and $\widetilde{D}_{q}(N)$ for the quantized standard map are similar to those for the CUE.

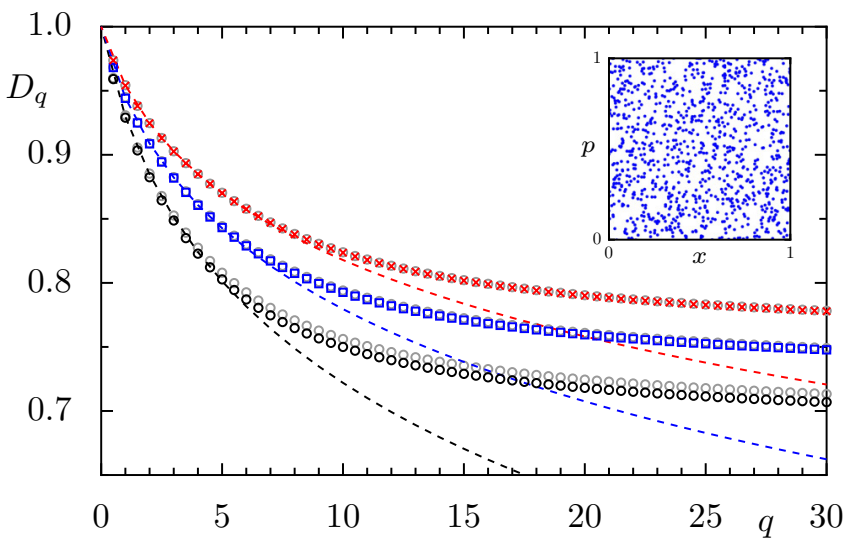

FIG. 5. Fractal dimensions $D_{q}(N)$ for the quantized standard map for $N=400,2000,10000$ (circles, squares, crosses) in comparison with $\widetilde{D}_{q}^{\mathrm{CUE}}(N)$, Eq. (33), dashed lines. Also shown are the CUE results of Fig. 2(a) as grey circles. The inset shows 1000 iterates of the standard map (49).

\section{MANY BODY SYSTEMS}

We now turn to another class of systems for which randommatrix theory is often applied: many-body systems which are neither integrable nor many-body-localized. We will present results for a specific spin chain (the XXZ chain with nearestneighbor interactions) for two different choices of parameters. In addition we have performed similar calculations for other many-body lattice Hamiltonians, and found the overall multifractality properties to be very similar. We thus believe the results presented here to be qualitatively generic.

\section{Hamiltonian}

We consider a disorder-free XXZ Heisenberg chain, consisting of $L$ sites and one spin-1/2 particle on each site, with both nearest-neighbor (NN) and next-nearest-neighbor (NNN) interactions:

$$
\begin{aligned}
H= & J_{1} \sum_{i=1}^{L-1}\left(S_{i}^{+} S_{i+1}^{-}+S_{i}^{-} S_{i+1}^{+}+\Delta_{1} S_{i}^{z} S_{i+1}^{z}\right) \\
& +J_{2} \sum_{i=2}^{L-2}\left(S_{i}^{+} S_{i+2}^{-}+S_{i}^{-} S_{i+2}^{+}+\Delta_{2} S_{i}^{z} S_{i+2}^{z}\right)
\end{aligned}
$$

Here $S_{i}^{ \pm}=S_{i}^{x} \pm \mathrm{i} S_{i}^{y}$ with $S_{i}^{x}=\frac{\hbar}{2} \sigma_{i}^{x}, S_{i}^{y}=\frac{\hbar}{2} \sigma_{i}^{y}$, and $S_{i}^{z}=\frac{\hbar}{2} \sigma_{i}^{z}$, using the Pauli-matrices acting only on the $i$-th site. The summations in (53) are over the site index. The XXZ chain with NNN interactions is a canonical example of a non-integrable many-body system. As such, the midspectrum eigenstates and the dynamics of this model and its variants have been studied from several perspectives in recent years (see, e.g, [34, 108-114]). Of course, the equilibrium (low-energy) properties of such models have been considered extensively, already in earlier decades, but these are less relevant to the present work. 

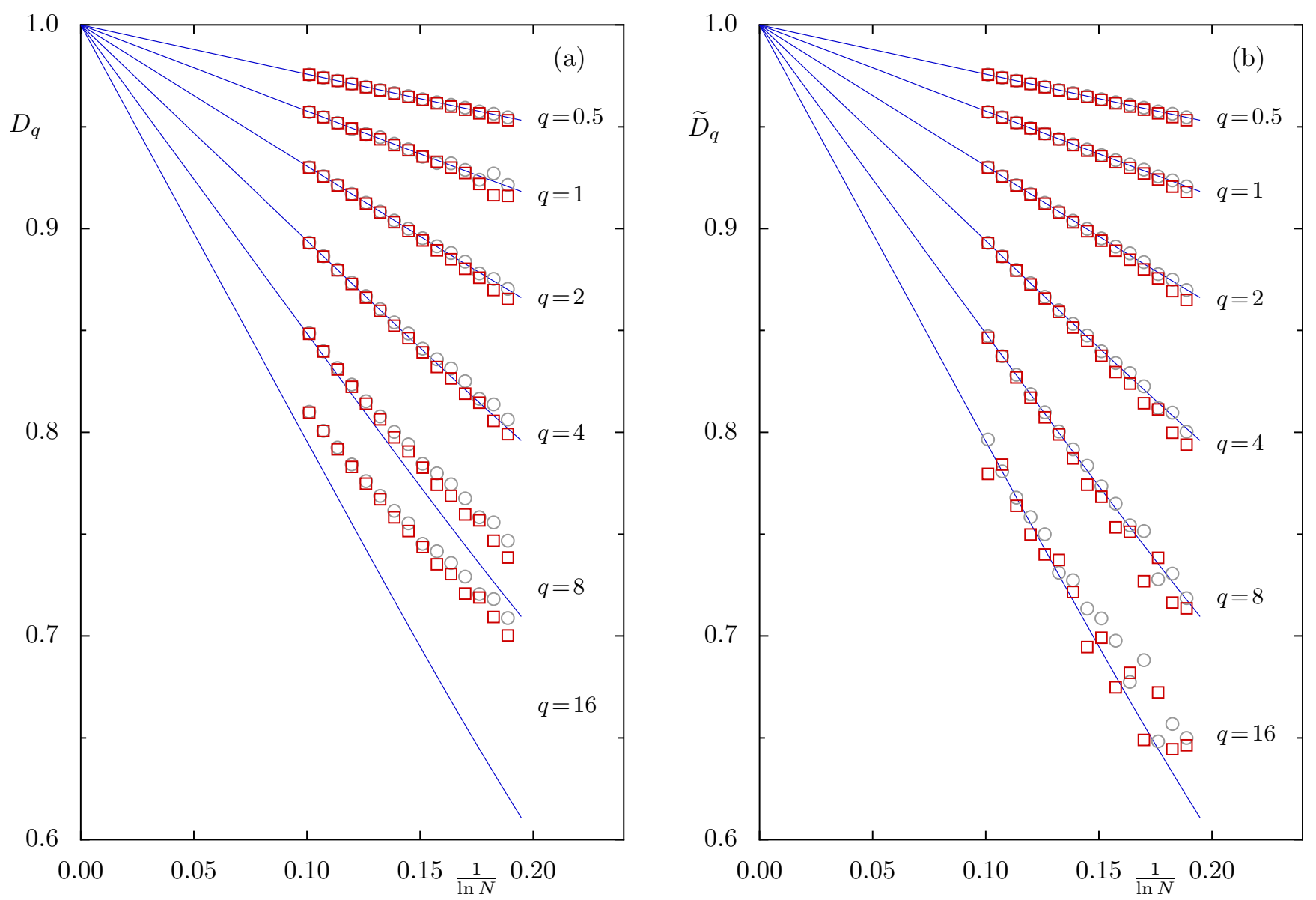

FIG. 6. Fractal dimensions (a) $D_{q}(N)$ and (b) $\widetilde{D}_{q}(N)$ for $q=0.5,1,2,4,8,16$ for the quantized standard map (red squares) vs. $1 /$ ln $N$. The full lines show $\widetilde{D}_{q}^{\text {CUE }}(N)$, Eq. (33). The grey circles show the corresponding random matrix results for one realization of the CUE.

In order to avoid reflection symmetry, we have omitted the NNN coupling between sites 1 and 3 (the summation starts from $i=2$ instead of $i=1$ ). The NNN coupling breaks integrability; to keep away from an integrable point we use $J_{2}=J_{1}$. We also set both couplings $J_{1,2}$ to unity, i.e., energies are measured in units of $J_{1}$.

The XXZ chain (53) conserves the total $S^{z}=\sum_{i=1}^{L-1} S_{i}^{z}$, or equivalently, the number of up-spins or "particle number" $M$. For $M$ up-spins in $L$ sites, the Hilbert space dimension is $N=\left(\begin{array}{c}L \\ M\end{array}\right)$. As parameters we use $\left(\Delta_{1}, \Delta_{2}\right)=(2.0,0.0)$ throughout the text, apart from Fig. 9, where in addition $\left(\Delta_{1}, \Delta_{2}\right)=(0.8,0.8)$ is used.

We have checked that the system shows the correct GOE level spacing statistics for either of these parameter sets, e.g., the average $\langle r\rangle$ of the ratio of successive consecutive-neighbor level spacings $[25,115]$ is near the value $(\approx 0.53)$ expected for the GOE. The ratio of the spacings between two closest levels $[116]$ is also near the GOE value $(\approx 0.57)$.

\section{Overview: various parts of the spectrum}

We first consider the fractal dimensions of all eigenstates for the XXZ spin chain (53) in the ergodic regime. Eigenstates at the very low-energy and very high-energy edges of the spectrum are multifractal. Indeed, for the lowest and the highest eigenstates $D_{q}^{\infty} \neq 1$ for $q \neq 0[34,60]$. For non-integrable systems, it is widely expected that the eigenstates in the middle of the many-body spectrum behave at least like randommatrix eigenstates; in fact this expectation may be considered the basic idea behind the eigenstate thermalization hypothesis [18-23, 113, 117-120]. Thus, we expect that the middle of the spectrum is at least weakly ergodic in the sense that the corresponding wavefunctions occupy a finite fraction of the Hilbert space and, thus, $D_{q}(N)$ approaches 1 in the $N \rightarrow \infty$ limit.

Figure 7 illustrates the $N$-dependence of the fractal dimensions by plotting $D_{2}(j, N)$ for every eigenstate $j$ of the nonintegrable spin-chain for different values of the Hilbert space dimension $N$ : As the system size increases, the $D_{q}(j, N)$ values for mid-spectrum eigenstates move up towards 1 , i.e. the eigenstates show the expected ergodic behavior. (We will later show that the approach to 1 is logarithmically slow.) In con- 


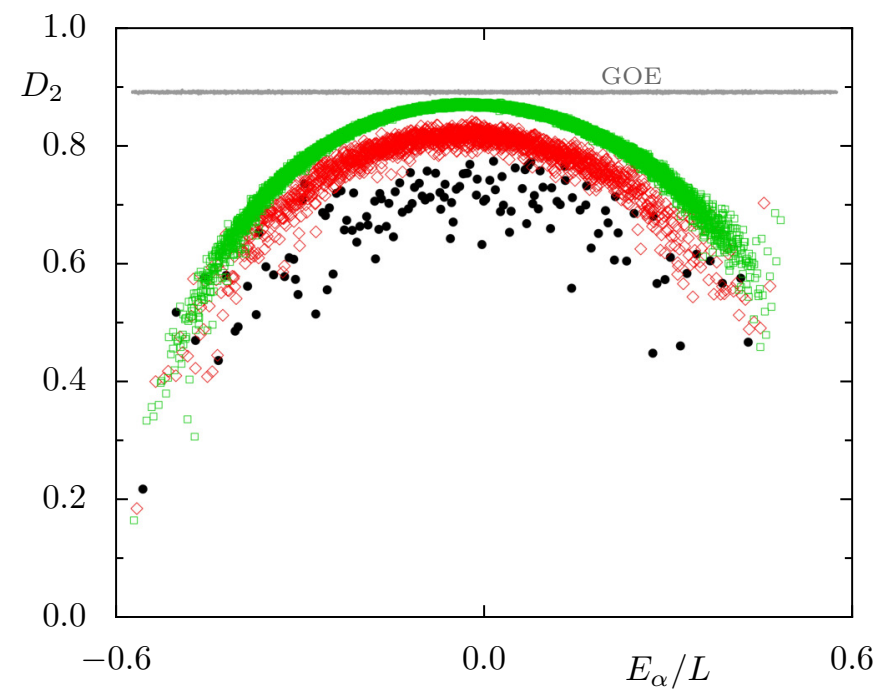

FIG. 7. Fractal dimensions $D_{q}(j, N)$ for $q=2$ vs. the scaled eigenenergies, $E_{\alpha} / L$, for the XXZ spin-chain. for $\left(L, n_{\uparrow}\right)=(9,4)$, $(13,6),(17,8)$ (black circles, red diamonds, green squares). The corresponding Hilbert space dimensions are $N=126,1716,24310$. For one realization of the GOE with $N=24310$ the fractal dimensions $D_{2}$ are shown as small grey dots versus the energies (rescaled to approximately the same bandwidth as the spin-chain).

trast, for the bottom or top of the spectrum there is no trend towards 1 which is consistent with the picture that these eigenstates are multifractal.

In contrast, the results for a realization of the GOE show no dependence on the energy, e.g. there is no multifractality near the edges of the spectrum, even though the spectral density of the GOE does depend on the energy. This is shown as the grey points forming a straight line in Fig. 7. For the purposes of the eigenvector statistics the results for the $\mathrm{COE}$ obtained in Sec. III A are identical to those of the GOE as only the normalization condition (12) is relevant. Also note that the results for the spin-chain (green squares) with the same dimension $N=24310$ are well below the GOE result, even in the middle of the spectrum. Therefore an important question, to be addressed in the next section, is how $D_{q}(N)$ approaches 1 for mid-spectrum many-body eigenstates.

\section{Comparison of the fractal dimensions with the GOE}

Figure 8 shows the $q$-dependence of the fractal dimensions $D_{q}(N)$ in the many-body system in comparison with numerical results for the GOE of the corresponding sizes $N$. To avoid fluctuations due to finite statistics we only show the typical fractal dimensions $D_{q}$. The overall shape and sizedependence is qualitatively similar to that in the COE, CUE, and standard map cases studied in previous sections. However, the departure from the random-matrix data is now much stronger: the deviations are already significant for the smallest moments and become more prominent with increasing $q$. With increasing system size $N$ the fractal dimensions $D_{q}(N)$

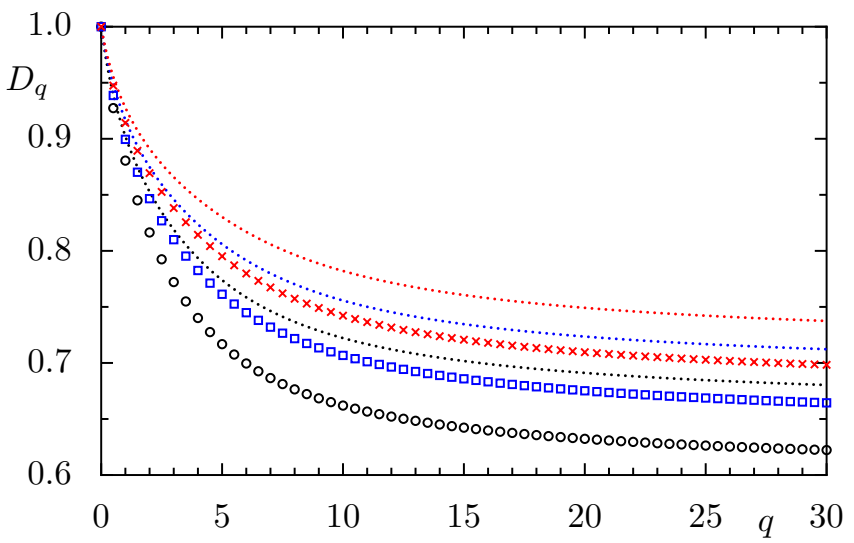

FIG. 8. Fractal dimensions $D_{q}(N)$ versus moments $q$ for the XXZ spin chain for different system sizes $\left(L, n_{\uparrow}\right)=(13,6),(15,7)$, $(17,8)$ (black circles, blue squares, red crosses) using 250 states in the middle of the spectrum. For comparison numerical results for realizations of the GOE results are shown as dotted curves of the correspondent colors for $N=1716,6435,24310$.

become larger, moving towards 1 , and the difference to the random matrix results becomes smaller.

The amount of the deviations from the COE prediction are highly system and parameter specific. Indeed, even considering the same many-body model (53) for either $\left(\Delta_{1}, \Delta_{2}\right)=$ $(2.0,0.0)$, as before, or $\left(\Delta_{1}, \Delta_{2}\right)=(0.8,0.8)$, reveals very different departures from the GOE results as illustrated in Fig. 9.

We have also examined the distribution $P\left(D_{q}\right)$ for the many-body eigenstates (not shown). The distribution is qualitatively similar to the one for the COE or CUE (which is shown in Fig. 3.)

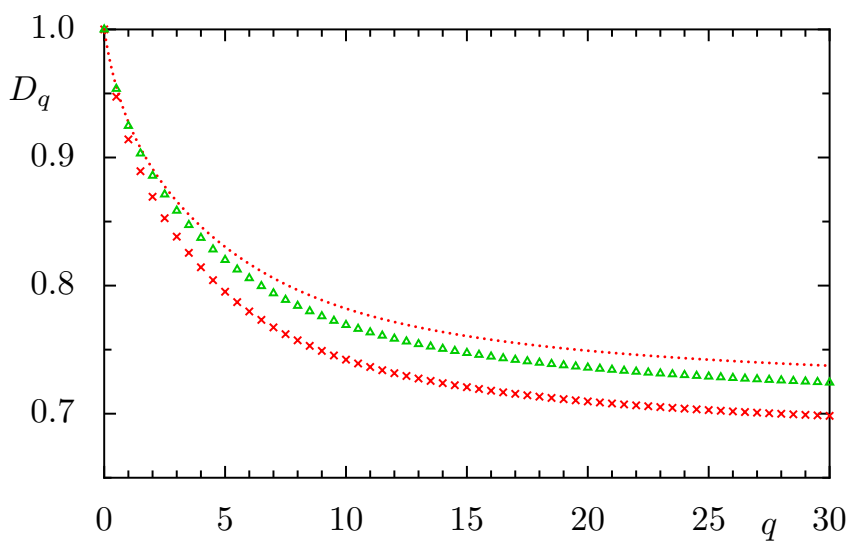

FIG. 9. Fractal dimensions $D_{q}(N)$ versus $q$ for the XXZ spin chain for $\left(L, n_{\uparrow}\right)=(17,8)$, such that $N=24310$, for $\left(\Delta_{1}, \Delta_{2}\right)=$ $(2.0,0.0)$, red crosses, as in Fig. 8 , and $\left(\Delta_{1}, \Delta_{2}\right)=(0.8,0.8)$, green triangles. In both cases 250 states in the middle of the spectrum are used. The red dotted line is the numerical result for one realization of the GOE for the corresponding matrix size $N=24310$, as in Fig. 8 . 


\section{Size dependence: weak ergodicity}

To systematically address the scaling limit of the fractal dimensions in the many-body system Fig. 10 shows $D_{q}(N)$ versus the $1 / \ln N$ (red squares), i.e. the inverse logarithm of the Hilbert space dimension, together with GOE data (grey circles) and the analytical prediction $\widetilde{D}_{q}^{\mathrm{COE}}(N)$, Eq. (21), (solid blue lines) for the corresponding matrix size $N$ for several moments $q$. The red dashed straight lines are guides to the eye connecting $D_{q}=1$ at $N \rightarrow \infty$ with the many-body data point at the largest considered system size. The error bars are given by the standard deviation of the distribution of the corresponding fractal dimensions $D_{q}(j, N)$.

It is clearly seen in Fig. 10 that the many-body data approaches $D_{q}(N) \rightarrow 1$ at $N \rightarrow \infty$, however the path of this approach is different from the one of the GOE: The fractal dimensions $D_{q}(N)$ are smaller, while the standard deviations (shown as error bars) are larger. This clearly suggests that the eigenstates of a typical non-integrable many-body system are only weakly ergodic, i.e., that they only occupy a finite fraction $\rho$ of the whole Hilbert space.

Following Eq. (9), we can express the weak ergodicity in terms of the scaling of the typical moments by comparing with the GOE result, Eq. (23),

$$
\left\langle I_{q}(j, N)\right\rangle_{\mathrm{typ}}=c_{q} N^{-D_{q}^{\infty}(q-1)}=c_{q}^{\mathrm{GOE}} N_{\mathrm{eff}}^{-D_{q}^{\infty}(q-1)} .
$$

where $N_{\text {eff }}=\rho N$ with fraction $\rho \equiv N_{\text {eff }} / N=$ $\left(c_{q}^{\mathrm{GOE}} / c_{q}\right)^{1 /\left(D_{q}^{\infty}(q-1)\right)}<1$. This shows that the deviation of $c_{q}$ compared to $c_{q}^{\mathrm{GOE}}$ corresponds to the effectively reduced fraction of the whole Hilbert space occupied by weaklyergodic eigenstates, compared to ergodic ones of the GOE.

The weak ergodicity also suggests that a standard random matrix ensemble like the GOE of GUE is not a fully correct description of the statistical properties of the many-body states, even in the middle of the spectrum. This can also be seen by examining the coefficient distribution. In the inset of Fig. 10, the distribution $P(\eta)$ of the (rescaled) eigenvector components $\eta=N\left|c_{i}^{(j)}\right|^{2}$ is shown. There are clear deviations from the Porter-Thomas distribution (19) of the GOE. The deviations at the tail of the distribution are highlighted here by using a logarithmic scale. Some deviations from the random-matrix expectation was also noted in Ref. [34].

\section{SUMMARY AND OUTLOOK}

In this work we have addressed the deviations of the eigenstate statistics from the fully ergodic result — for random matrix ensembles, a single-particle system with chaotic classical dynamics, and chaotic many-body systems. We analyzed the scaling behavior of the fractal dimensions $D_{q}(N)$ which should approach one in the limit of large system size $N$ if the system is fully ergodic.

For the standard random matrix ensembles (COE and CUE) we provide analytical results for the means $\widetilde{D}_{q}(N)$ over individual eigenstates. This provides a lower bound for the typical $D_{q}(N)$ (logarithmic) averages of eigenstate moments. We

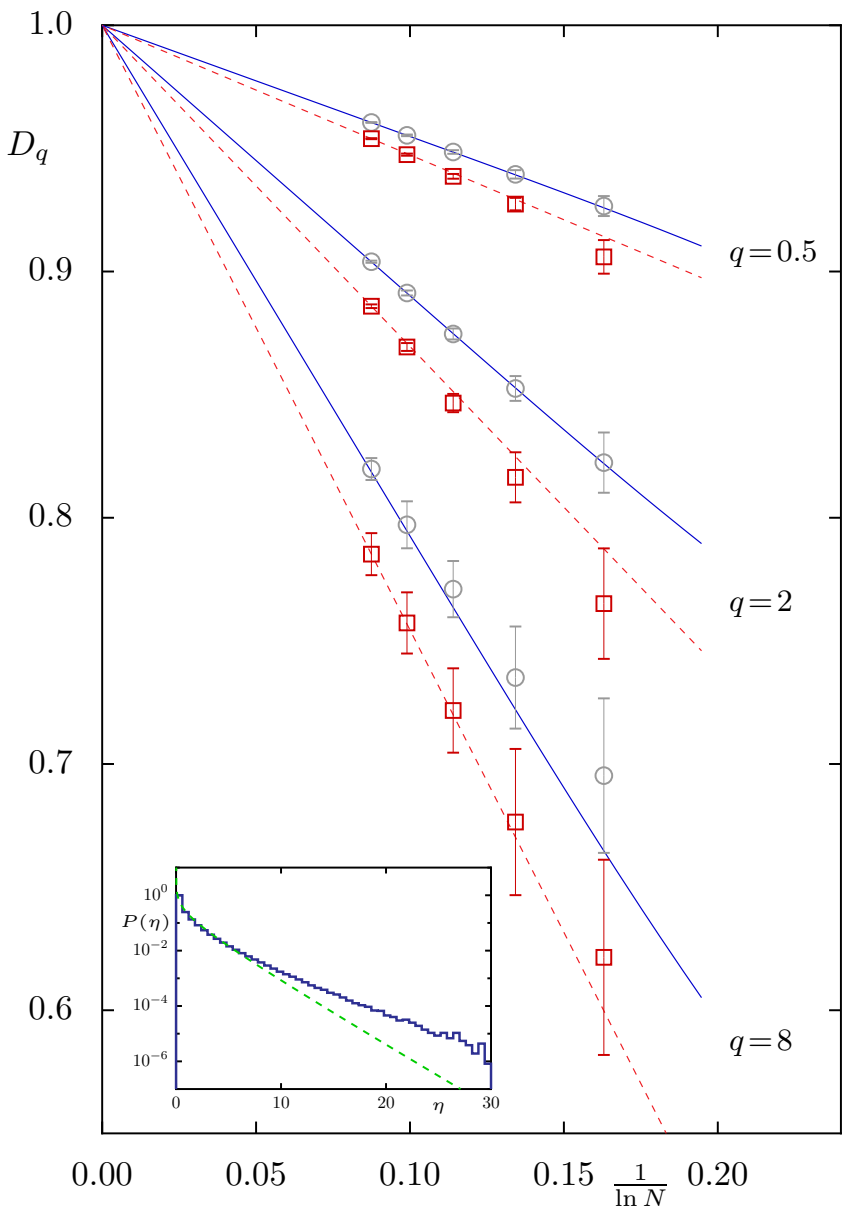

FIG. 10. Fractal dimensions $D_{q}(N)$ for $q=0.5,2,8$ for the XXZ spin-chain (red squares) versus $1 / \ln N$ for the sequence of system sizes $\left(L, n_{\uparrow}\right)=(11,5),(13,6),(15,7),(17,8),(19,9)$. The 250 states in the middle of the spectrum are used for all sizes except the largest one (for $\left(L, n_{\uparrow}\right)=(19,9) 150$ states are used). The solid blue lines show the COE analytical prediction $\widetilde{D}_{q}^{\mathrm{COE}}(N)$, Eq. (21). The grey circles show the corresponding random matrix results for one realization of the GOE for $N=462,1716,6435,24310$, 92378. The vertical bars indicate the standard deviation of the data. The red dashed lines for each $q$ are guides to the eye connecting $\left(1 / \ln N, D_{q}(N)\right)=(0,1)$ with the value of $D_{q}(N)$ at the largest available system size (data point with smallest value of $1 / \ln N$ ). The inset shows the coefficient distribution $P(\eta)$ of the 250 eigenvectors for $\left(L, n_{\uparrow}\right)=(17,8)$ in a semi-logarithmic representation. Clear deviations from the Porter-Thomas distribution (19) of the GOE (green dashed line) are found.

show that individual realizations of COE and CUE typically match the predictions only for small $q$, and deviate at larger $q$ due to finite statistics. We have provided an estimate of the value $q^{*}(N)$ beyond which finite-statistics effects become important: $q^{*}(N)$ scales logarithmically with $N$ such that obtaining agreement at larger $q$ would require averaging over an exponentially large number of realizations.

For the quantized standard map with classically chaotic dynamics, the numerical results agree well with those for realizations of random matrices. For both random matrices and 
the quantized standard map, the approach $D_{q}(N) \rightarrow 1$ with increasing system size is slow, and closely follows the form $D_{q}^{\infty}-f_{q} / \ln N$ for small $q$. For larger $q$, there are strong deviations from this form and the data even shows some curvature when $D_{q}(N)$ is plotted against $1 / \ln N$. This curvature implies an $N$-dependence of the quantity $f_{q}$, or equivalently, of the quantity $c_{q}=\mathrm{e}^{(q-1) f_{q}}$ used in Eq. (9).

In contrast, the results for the many-body systems deviate quite significantly from the $\mathrm{COE}$ data. We have analyzed these deviations in $D_{q}(N)$ for different eigenstates, different values of $q$, and system sizes. The fractal dimensions of the non-integrable many-body systems still approach the ergodic limit $D_{q}(N)=1$ in the thermodynamic limit $N \rightarrow \infty$. However, the path of this approach differs from the randommatrix one and is system-specific: writing the $N$-dependence as $D_{q}^{\infty}-f_{q} / \ln N$ requires $c_{q}$ to be larger than the GOE value $c_{q}^{\mathrm{GOE}}$. We thus conclude that mid-spectrum many-body eigenstates are of weakly ergodic nature and occupy only a finite fraction of the whole Hilbert space. We speculate that this may result from the fact that mid-spectrum eigenstates are forced to be orthogonal to the eigenstates at the spectral edges, which are very special (multifractal).

The present work opens up various new questions. (1) The curvature in the $D_{q}(N)$ versus $1 / \ln N$ plots points to finitesize structures in random-matrix eigenstates which deserve further study. If we write $D_{q}(N)$ as $D_{q}^{\infty}-f_{q} / \ln N$, then $c_{q}=\mathrm{e}^{(q-1) f_{q}}$ is weakly $N$-dependent; the form of depen- dence is a non-trivial characterization of finite-size randommatrix eigenstates which would be interesting to investigate. (2) We have characterized multifractality properties of the quantized standard map at large $K$, for which the classical counterpart is strongly chaotic. As $K$ is decreased, the classical dynamics shows a mixed phase space in which regular motion and chaotic motion coexist on arbitrarily fine scales. This will change the behavior of $D_{q}(N)$ and could lead to weak ergodicity or multifractality. (3) We have only examined manybody models which are nominally chaotic. The $D_{q}(N)$ behaviors of mid-spectrum eigenstates of integrable many-body systems remains an open issue. (4) Our results suggest that many-body eigenstates are only weakly ergodic. This implies that the standard random matrix classes (GOE, GUE) may not be the optimal random-matrix models for describing the mid-spectrum eigenstates. Other random matrix classes, such as the power-law-banded random matrices [17, 38, 97, 121123], might be fruitful to examine as models of eigenstates of non-integrable many-body Hamiltonians.

\section{ACKNOWLEDGMENTS}

We thank Wouter Beugeling and Paul McClarty for useful discussions. I. M. K. acknowledges the support of German Research Foundation (DFG) Grant No. KH 425/1-1 and the Russian Foundation for Basic Research Grant No. 17-5212044.
[1] T. A. Brody, J. Flores, J. B. French, P. A. Mello, A. Pandey, and S. S. M. Wong, Random-matrix physics: spectrum and strength fluctuations, Rev. Mod. Phys. 53, 385 (1981).

[2] M. L. Mehta, Random Matrices, Elsevier Ltd., 3rd edition (2004).

[3] M. V. Berry, Regular and irregular semiclassical wavefunctions, J. Phys. A 10, 2083 (1977).

[4] M. Shapiro and G. Goelman, Onset of chaos in an isolated energy eigenstate, Phys. Rev. Lett. 53, 1714 (1984).

[5] S. W. McDonald and A. N. Kaufman, Wave chaos in the stadium: Statistical properties of short-wave solutions of the Helmholtz equation, Phys. Rev. A 37, 3067 (1988).

[6] R. Aurich and F. Steiner, Exact theory for the quantum eigenstates of a strongly chaotic system, Physica D 48, 445 (1991).

[7] R. Aurich and F. Steiner, Statistical properties of highly excited quantum eigenstates of a strongly chaotic system, Physica D 64, 185 (1993).

[8] B. Li and M. Robnik, Statistical properties of high-lying chaotic eigenstates, J. Phys. A 27, 5509 (1994).

[9] F. Simmel and M. Eckert, Statistical measures for eigenfunctions of nonseparable quantum billiard systems, Physica D 97, 517 (1996).

[10] T. Prosen, Quantization of generic chaotic 3D billiard with smooth boundary II: Structure of high-lying eigenstates, Phys. Lett. A 233, 332 (1997).

[11] A. Bäcker, Numerical aspects of eigenvalues and eigenfunctions of chaotic quantum systems, in M. Degli Esposti and
S. Graffi (editors) "The Mathematical Aspects of Quantum Maps”, volume 618 of Lect. Notes Phys., 91, Springer-Verlag, Berlin (2003).

[12] F. M. Izrailev, Chaotic stucture of eigenfunctions in systems with maximal quantum chaos, Phys. Lett. A 125, 250 (1987).

[13] S. Nonnenmacher, Crystal properties of eigenstates for quantum cat maps, Nonlinearity 10, 1569 (1997).

[14] M. Kuś, J. Mostowski, and F. Haake, Universality of eigenvector statistics of kicked tops of different symmetries, J. Phys. A 21, L1073 (1988).

[15] S. Nonnenmacher and A. Voros, Chaotic eigenfunctions in phase space, J. Stat. Phys. 92, 431 (1998).

[16] S. Nonnenmacher, Anatomy of quantum chaotic eigenstates, Séminaire Poincaré XIV, 177 (2010).

[17] F. Evers and A. D. Mirlin, Anderson transitions, Rev. Mod. Phys. 80, 1355 (2008).

[18] J. M. Deutsch, Quantum statistical mechanics in a closed system, Phys. Rev. A 43, 2046 (1991).

[19] M. Srednicki, Chaos and quantum thermalization, Phys. Rev. E 50, 888 (1994).

[20] M. Rigol, V. Dunjko, and M. Olshanii, Thermalization and its mechanism for generic isolated quantum systems, Nature 452, 854 (2008).

[21] A. Polkovnikov, K. Sengupta, A. Silva, and M. Vengalattore, Colloquium: Nonequilibrium dynamics of closed interacting quantum systems, Rev. Mod. Phys. 83, 863 (2011). 
[22] L. D’Alessio, Y. Kafri, A. Polkovnikov, and M. Rigol, From quantum chaos and eigenstate thermalization to statistical mechanics and thermodynamics, Adv. Phys. 65, 239 (2016).

[23] F. Borgonovi, F. M. Izrailev, L. F. Santos, and V. G. Zelevinsky, Quantum chaos and thermalization in isolated systems of interacting particles, Phys. Rep. 626, 1 (2016).

[24] D. M. Basko, I. L. Aleiner, and B. L. Altshuler, Metalinsulator transition in a weakly interacting many-electron system with localized single-particle states, Ann. Phys. (N.Y.) 321, 1126 (2006).

[25] V. Oganesyan and D. A. Huse, Localization of interacting fermions at high temperature, Phys. Rev. B 75, 155111 (2007).

[26] R. Nandkishore and D. A. Huse, Many-body localization and thermalization in quantum statistical mechanics, Annu. Rev. Condens. Matter Phys. 6, 15 (2015).

[27] E. Altman and R. Vosk, Universal dynamics and renormalization in many-body-localized systems, Annu. Rev. Condens. Matter Phys. 6, 383 (2015).

[28] J. Z. Imbrie, On many-body localization for quantum spin chains, J. Stat. Phys. 163, 998 (2016).

[29] M. Serbyn, Z. Papić, and D. A. Abanin, Thouless energy and multifractality across the many-body localization transition, Phys. Rev. B 96, 104201 (2017).

[30] D. J. Luitz and Y. Bar Lev, The ergodic side of the many-body localization transition, Ann. Phys. 529, 1600350 (2017).

[31] F. Alet and N. Laflorencie, Many-body localization: An introduction and selected topics, C. R. Physique 19, 498 (2018).

[32] A. Bäcker and R. Schubert, Amplitude distribution of eigenfunctions in mixed systems, J. Phys. A 35, 527 (2002).

[33] A. Bäcker, Random waves and more: Eigenfunctions in chaotic and mixed systems, The European Physical Journal Special Topics 145, 161 (2007).

[34] W. Beugeling, A. Bäcker, R. Moessner, and M. Haque, Statistical properties of eigenstate amplitudes in complex quantum systems, Phys. Rev. E 98, 022204 (2018).

[35] R. Samajdar and S. R. Jain, Exact eigenfunction amplitude distributions of integrable quantum billiards, J. Math. Phys. 59, 012103 (2018).

[36] A. De Luca and A. Scardicchio, Ergodicity breaking in a model showing many-body localization, Europhys. Lett. 101, 37003 (2013).

[37] E. Bogomolny and M. Sieber, Eigenfunction distribution for the Rosenzweig-Porter model, Phys. Rev. E 98, 032139 (2018).

[38] E. Bogomolny and M. Sieber, Power-law random banded matrices and ultrametric matrices: Eigenvector distribution in the intermediate regime, Phys. Rev. E 98, 042116 (2018).

[39] K. Truong and A. Ossipov, Statistical properties of eigenvectors and eigenvalues of structured random matrices, J. Phys. A 51, 065001 (2018).

[40] R. Aurich, A. Bäcker, R. Schubert, and M. Taglieber, Maximum norms of chaotic quantum eigenstates and random waves, Physica D 129, 1 (1999).

[41] J. T. Edwards and D. J. Thouless, Numerical studies of localization in disordered systems, J. Phys. C 5, 807 (1972).

[42] $\mathrm{F}$. Wegner, Inverse participation ratio in $2+\epsilon$ dimensions, Z. Phys. B 36, 209 (1980).

[43] B. Kramer and A. MacKinnon, Localization: Theory and experiment, Rep. Prog. Phys. 56, 1469 (1993).

[44] F. Evers and A. D. Mirlin, Fluctuations of the inverse participation ratio at the Anderson transition, Phys. Rev. Lett. 84, 3690 (2000).

[45] L. F. Santos and M. Rigol, Onset of quantum chaos in onedimensional bosonic and fermionic systems and its relation to thermalization, Phys. Rev. E 81, 036206 (2010).

[46] W. Beugeling, A. Andreanov, and M. Haque, Global characteristics of all eigenstates of local many-body Hamiltonians: participation ratio and entanglement entropy, J. Stat. Mech. 2015, P02002 (2015).

[47] D. J. Luitz, N. Laflorencie, and F. Alet, Many-body localization edge in the random-field Heisenberg chain, Phys. Rev. B 91, 081103(R) (2015).

[48] E. J. Torres-Herrera and L. F. Santos, Dynamics at the manybody localization transition, Phys. Rev. B 92, 014208 (2015).

[49] G. Misguich, V. Pasquier, and J.-M. Luck, Inverse participation ratios in the XXZ spin chain, Phys. Rev. B 94, 155110 (2016).

[50] E. Tsukerman, Inverse participation ratios in the $X X$ spin chain, Phys. Rev. B 95, 115121 (2017).

[51] X. Jia, A. R. Subramaniam, I. A. Gruzberg, and S. Chakravarty, Entanglement entropy and multifractality at localization transitions, Phys. Rev. B 77, 014208 (2008).

[52] O. Giraud, J. Martin, and B. Georgeot, Entropy of entanglement and multifractal exponents for random states, Phys. Rev. A 79, 032308 (2009).

[53] J.-M. Stéphan, S. Furukawa, G. Misguich, and V. Pasquier, Shannon and entanglement entropies of one- and twodimensional critical wave functions, Phys. Rev. B 80, 184421 (2009).

[54] J.-M. Stéphan, G. Misguich, and V. Pasquier, Rényi entropy of a line in two-dimensional Ising models, Phys. Rev. B 82, 125455 (2010).

[55] L. F. Santos and M. Rigol, Localization and the effects of symmetries in the thermalization properties of one-dimensional quantum systems, Phys. Rev. E 82, 031130 (2010).

[56] J.-M. Stéphan, G. Misguich, and V. Pasquier, Phase transition in the Rényi-Shannon entropy of Luttinger liquids, Phys. Rev. B 84, 195128 (2011).

[57] D. J. Luitz, F. Alet, and N. Laflorencie, Universal behavior beyond multifractality in quantum many-body systems, Phys. Rev. Lett. 112, 057203 (2014).

[58] H. G. E. Hentschel and I. Procaccia, The infinite number of generalized dimensions of fractals and strange attractors, Physica D 8, 435 (1983).

[59] T. C. Halsey, M. H. Jensen, L. P. Kadanoff, I. Procaccia, and B. I. Shraiman, Fractal measures and their singularities: The characterization of strange sets, Phys. Rev. A 33, 1141 (1986).

[60] Y. Y. Atas and E. Bogomolny, Multifractality of eigenfunctions in spin chains, Phys. Rev. E 86, 021104 (2012).

[61] N. Macé, F. Alet, and N. Laflorencie, Multifractal scalings across the many-body localization transition, arXiv:1812.10283 [cond-mat.dis-nn] (2018).

[62] O. Bohigas, M. J. Giannoni, and C. Schmit, Characterization of chaotic quantum spectra and universality of level fluctuation laws, Phys. Rev. Lett. 52, 1 (1984).

[63] T. Guhr, A. Müller-Groeling, and H. A. Weidenmüller, Random-matrix theories in quantum physics: common concepts, Phys. Rep. 299, 189 (1998).

[64] D. Ullmo, Bohigas-Giannoni-Schmit conjecture, Scholarpedia 11, 31721 (2016).

[65] E. Bogomolny and O. Giraud, Perturbation approach to multifractal dimensions for certain critical random-matrix ensembles, Phys. Rev. E 84, 036212 (2011).

[66] E. Bogomolny and O. Giraud, Multifractal dimensions for all moments for certain critical random-matrix ensembles in the strong multifractality regime, Phys. Rev. E 85, 046208 (2012).

[67] J. A. Méndez-Bermúdez, A. Alcazar-López, and I. Varga, On the generalized dimensions of multifractal eigenstates, 
J. Stat. Mech. 2014, P11012 (2014).

[68] R. Dubertrand, I. García-Mata, B. Georgeot, O. Giraud, G. Lemarié, and J. Martin, Two scenarios for quantum multifractality breakdown, Phys. Rev. Lett. 112, 234101 (2014).

[69] L. Ujfalusi and I. Varga, Finite-size scaling and multifractality at the Anderson transition for the three Wigner-Dyson symmetry classes in three dimensions, Phys. Rev. B 91, 184206 (2015).

[70] R. Dubertrand, I. García-Mata, B. Georgeot, O. Giraud, G. Lemarié, and J. Martin, Multifractality of quantum wave functions in the presence of perturbations, Phys. Rev. E 92, 032914 (2015).

[71] J. Lindinger and A. Rodríguez, Multifractal finite-size scaling at the Anderson transition in the unitary symmetry class, Phys. Rev. B 96, 134202 (2017).

[72] A. De Luca, B. L. Altshuler, V. E. Kravtsov, and A. Scardicchio, Anderson localization on the Bethe lattice: Nonergodicity of extended states, Phys. Rev. Lett. 113, 046806 (2014).

[73] B. L. Altshuler, E. Cuevas, L. B. Ioffe, and V. E. Kravtsov, Nonergodic phases in strongly disordered random regular graphs, Phys. Rev. Lett. 117, 156601 (2016).

[74] V. E. Kravtsov, B. L. Altshuler, and L. B. Ioffe, Non-ergodic delocalized phase in Anderson model on Bethe lattice and regular graph, Ann. Phys. 389, 148 (2018).

[75] V. E. Kravtsov, I. M. Khaymovich, E. Cuevas, and M. Amini, A random matrix model with localization and ergodic transitions, New J. Phys. 17, 122002 (2015).

[76] M. Pino, V. E. Kravtsov, B. L. Altshuler, and L. B. Ioffe, Multifractal metal in a disordered Josephson junctions array, Phys. Rev. B 96, 214205 (2017).

[77] S. Roy, I. Khaymovich, A. Das, and R. Moessner, Multifractality without fine-tuning in a Floquet quasiperiodic chain, SciPost Phys. 4, 025 (2018).

[78] S. Bera, G. De Tomasi, I. M. Khaymovich, and A. Scardicchio, Return probability for the Anderson model on the random regular graph, Phys. Rev. B 98, 134205 (2018).

[79] G. De Tomasi, M. Amini, S. Bera, I. Khaymovich, and V. Kravtsov, Survival probability in generalized RosenzweigPorter random matrix ensemble, SciPost Phys. 6, 014 (2019).

[80] P. A. Nosov, I. M. Khaymovich, and V. E. Kravtsov, Correlation-induced localization, Phys. Rev. B 99, 104203 (2019).

[81] V. N. Smelyanskiy, K. Kechedzhi, S. Boixo, S. V. Isakov, H. Neven, and B. Altshuler, Non-ergodic delocalized states for efficient population transfer within a narrow band of the energy landscape, arXiv:1802.09542 [quant-ph] (2018).

[82] K. Kechedzhi, V. Smelyanskiy, J. R. McClean, V. S. Denchev, M. Mohseni, S. Isakov, S. Boixo, B. Altshuler, and H. Neven, Efficient population transfer via non-ergodic extended states in quantum spin glass, arXiv:1807.04792 [quant-ph] (2018).

[83] L. Faoro, M. Feigel'man, and L. Ioffe, Non-ergodic phase of the quantum random energy model, arXiv:1812.06016 [condmat.dis-nn] (2018).

[84] T. Micklitz, F. Monteiro, and A. Altland, Non-ergodic extended states in the SYK model, arXiv:1901.02389 [condmat.str-el] (2019).

[85] M. Pino, J. Tabanera, and P. Serna, From ergodic to nonergodic chaos in Rosenzweig-Porter model, arXiv:1904.02716 [cond-mat.dis-nn] (2019).

[86] P. A. Nosov and I. M. Khaymovich, Immunity of delocalization to soft constraints in long-range random models, arXiv:1904.11509 [cond-mat.dis-nn] (2019).

[87] J. Martin, O. Giraud, and B. Georgeot, Multifractality and intermediate statistics in quantum maps, Phys. Rev. E 77,
035201(R) (2008).

[88] J. Martin, I. García-Mata, O. Giraud, and B. Georgeot, Multifractal wave functions of simple quantum maps, Phys. Rev. E 82, 046206 (2010).

[89] Y. Y. Atas and E. Bogomolny, Calculation of multi-fractal dimensions in spin chains, Phil. Trans. R. Soc. A 372, 20120520 (2014).

[90] J. Lindinger and A. Rodríguez, Multifractality in Fock space of the ground state of the Bose-Hubbard Hamiltonian, Acta Physica Polonica A 132, 1683 (2017).

[91] J. Lindinger, A. Buchleitner, and A. Rodríguez, Many-body multifractality throughout bosonic superfluid and Mott insulator phases, Phys. Rev. Lett. 122, 106603 (2019).

[92] T. L. M. Lezama, S. Bera, and J. H. Bardarson, Apparent slow dynamics in the ergodic phase of a driven manybody localized system without extensive conserved quantities, Phys. Rev. B 99, 161106(R) (2019).

[93] K. S. Tikhonov and A. D. Mirlin, Fractality of wave functions on a Cayley tree: Difference between tree and locally treelike graph without boundary, Phys. Rev. B 94, 184203 (2016).

[94] M. Sonner, K. S. Tikhonov, and A. D. Mirlin, Multifractality of wave functions on a Cayley tree: From root to leaves, Phys. Rev. B 96, 214204 (2017).

[95] K. S. Tikhonov, A. D. Mirlin, and M. A. Skvortsov, Anderson localization and ergodicity on random regular graphs, Phys. Rev. B 94, 220203(R) (2016).

[96] G. Biroli and M. Tarzia, Delocalized glassy dynamics and many-body localization, Phys. Rev. B 96, 201114(R) (2017).

[97] A. D. Mirlin and F. Evers, Multifractality and critical fluctuations at the Anderson transition, Phys. Rev. B 62, 7920 (2000).

[98] C. E. Porter and R. G. Thomas, Fluctuations of nuclear reaction widths, Phys. Rev. 104, 483 (1956).

[99] NIST Digital library of mathematical functions, http://dlmf.nist.gov/, Release 1.0.21 of 2018-12-15, F. W. J. Olver, A. B. Olde Daalhuis, D. W. Lozier, B. I. Schneider, R. F. Boisvert, C. W. Clark, B. R. Miller and B. V. Saunders, (eds.).

[100] F. Mezzadri, How to generate random matrices from the classical compact groups, Not. Am. Math. Soc. 54, 592 (2007).

[101] B. V. Chirikov, A universal instability of many-dimensional oscillator systems, Phys. Rep. 52, 263 (1979).

[102] A. Gorodetski, On stochastic sea of the standard map, Commun. Math. Phys. 309, 155 (2012).

[103] M. V. Berry, N. L. Balazs, M. Tabor, and A. Voros, Quantum maps, Ann. Phys. (N.Y.) 122, 26 (1979).

[104] J. H. Hannay and M. V. Berry, Quantization of linear maps on a torus - Fresnel diffraction by a periodic grating, Physica D 1, 267 (1980).

[105] S.-J. Chang and K.-J. Shi, Evolution and exact eigenstates of a resonant quantum system, Phys. Rev. A 34, 7 (1986).

[106] J. P. Keating, F. Mezzadri, and J. M. Robbins, Quantum boundary conditions for torus maps, Nonlinearity 12, 579 (1999).

[107] M. Degli Esposti and S. Graffi, Mathematical aspects of quantum maps, in "The Mathematical Aspects of Quantum Maps", volume 618 of Lect. Notes Phys., 49, Springer-Verlag, Berlin (2003).

[108] L. F. Santos, Transport and control in one-dimensional systems, J. Math. Phys. 50, 095211 (2009).

[109] L. F. Santos, F. Borgonovi, and F. M. Izrailev, Onset of chaos and relaxation in isolated systems of interacting spins: Energy shell approach, Phys. Rev. E 85, 036209 (2012).

[110] R. Steinigeweg, J. Herbrych, and P. Prelovšek, Eigenstate thermalization within isolated spin-chain systems, 
Phys. Rev. E 87, 012118 (2013).

[111] P. M. Poggi and D. A. Wisniacki, Optimal control of manybody quantum dynamics: Chaos and complexity, Phys. Rev. A 94, 033406 (2016).

[112] E. Torres-Herrera, J. Karp, M. Távora, and L. Santos, Realistic many-body quantum systems vs. full random matrices: Static and dynamical properties, Entropy 18, 359 (2016).

[113] I. M. Khaymovich, M. Haque, and P. A. McClarty, Eigenstate thermalization, random matrix theory, and behemoths, Phys. Rev. Lett. 122, 070601 (2019).

[114] F. Borgonovi, F. M. Izrailev, and L. F. Santos, Exponentially fast dynamics of chaotic many-body systems, Phys. Rev. E 99, 010101(R) (2019).

[115] Y. Y. Atas, E. Bogomolny, O. Giraud, and G. Roux, Distribution of the ratio of consecutive level spacings in random matrix ensembles, Phys. Rev. Lett. 110, 084101 (2013).

[116] S. C. L. Srivastava, A. Lakshminarayan, S. Tomsovic, and A. Bäcker, Ordered level spacing probability densities, J. Phys. A 52, 025101 (2019).

[117] C. Neuenhahn and F. Marquardt, Thermalization of interacting fermions and delocalization in fock space, Phys. Rev. E 85,
060101(R) (2012).

[118] W. Beugeling, R. Moessner, and M. Haque, Finite-size scaling of eigenstate thermalization, Phys. Rev. E 89, 042112 (2014).

[119] P. Reimann, Eigenstate thermalization: Deutsch's approach and beyond, New J. Phys. 17, 055025 (2015).

[120] C. Nation and D. Porras, Off-diagonal observable elements from random matrix theory: Distributions, fluctuations, and eigenstate thermalization, New J. Phys. 20, 103003 (2018).

[121] A. D. Mirlin, Y. V. Fyodorov, F.-M. Dittes, J. Quezada, and T. H. Seligman, Transition from localized to extended eigenstates in the ensemble of power-law random banded matrices, Phys. Rev. E 54, 3221 (1996).

[122] V. E. Kravtsov and K. A. Muttalib, New class of random matrix ensembles with multifractal eigenvectors, Phys. Rev. Lett. 79, 1913 (1997).

[123] I. Varga and D. Braun, Critical statistics in a power-law random-banded matrix ensemble, Phys. Rev. B 61, R11859 (2000). 